\title{
Long term survival of cytoreductive surgery plus hyperthermic intraperitoneal chemotherapy in advanced epithelial ovarian cancer
}

\author{
Jue Zhang ${ }^{1}$, Xin-Bao $\mathrm{Li}^{1}$, Ru Ma ${ }^{1}$, Zhong-He Ji ${ }^{1}$, Wenpei Bai ${ }^{2}$, Yan $\mathrm{Li}^{1}$ \\ ${ }^{1}$ Department of Peritoneal Cancer Surgery, Beijing Shijitan Hospital, Capital Medical University, Beijing, China; ${ }^{2}$ Department of Gynecology, \\ Beijing Shijitan Hospital, Capital Medical University, Beijing, China \\ Contributions: (I) Conception and design: J Zhang, Y Li; (II) Administrative support: W Bai, Y Li; (III) Provision of study materials or patients: Y Li, \\ XB Li; (IV) Collection and assembly of data: R Ma, ZH Ji; (V) Data analysis and interpretation: J Zhang; (VI) Manuscript writing: All authors; (VII) \\ Final approval of manuscript: All authors. \\ Correspondence to: Yan Li, MD, PhD. Department of Peritoneal Cancer Surgery, Beijing Shijitan Hospital, Capital Medical University, No. 10 Tieyi \\ Road, Yangfangdian Street, Haidian District, Beijing 100038, China. Email: liyansd2@163.com.
}

\begin{abstract}
Background: To compare the efficacy of conventional debulking surgery and cytoreductive surgery (CRS) plus hyperthermic intraperitoneal chemotherapy (HIPEC) in patients with the peritoneal metastasis of epithelial ovarian cancer (EOCPC).
\end{abstract}

Methods: In patients with ovarian cancer who underwent surgery-based multidisciplinary treatment at our center from May 2004 to November 2019, 186 EOCPC patients were divided into a control group (conventional debulking surgery, $\mathrm{n}=115$ ) and a study group (CRS+HIPEC, $\mathrm{n}=71$ ) and were matched for baseline characteristics by propensity score matching (PSM). The endpoints were median overall survival (mOS) and median progression-free survival (mPFS).

Results: After matching, 133 patients met the selection criteria, including 80 patients in the control group and 53 patients in the study group. The mOS in the study group was significantly longer than that in the control group (87.3 vs. 25.2 months, respectively, $\mathrm{P}=0.002$ ). For complete cytoreduction, the mPFS in the study group was significantly longer than that in the control group [(19.6 vs. 10.1 months, respectively, $\mathrm{P}=0.007)$ ]. For complete CRS, the mOS in the study group was significantly longer than that in the control group [103.3 vs. 46.2 months, respectively, $\mathrm{P}=0.020]$. For incomplete CRS, the mOS in the study group was not different between the two groups.

Conclusions: Standardized CRS+HIPEC can contribute significant survival benefits to patients with EOCPC.

Keywords: Epithelial ovarian cancer (EOC); peritoneal carcinomatosis (PC); CRS+HIPEC; propensity score matching (PSM); survival

Submitted Nov 09, 2020. Accepted for publication Jul 05, 2021.

doi: $10.21037 /$ tcr-20-3233

View this article at: https://dx.doi.org/10.21037/tcr-20-3233

\section{Introduction}

The majority of patients with epithelial ovarian cancer (EOC) are diagnosed at the stage of advanced disease that has spread beyond the ovaries to cause peritoneal carcinomatosis (PC), which accounts for the highest mortality of all gynecologic cancers $(1,2)$. Even after the standard treatment of advanced EOC, in which optimal debulking surgery is usually followed by intravenous platinum/taxane-based chemotherapy (3), $75 \%$ of the patients still develop recurrence and present with PC (4), which is the most difficult obstacle to EOC treatment progress. 
Over the past three decades, aggressive cytoreductive surgery (CRS) plus hyperthermic intraperitoneal chemotherapy (HIPEC) has been developed as a comprehensive treatment package integrating multivesicular resections to remove macroscopic residual tumors, and HIPEC eradicates residual cancer cells after CRS. As a new treatment strategy for EOCPC, the efficacy of CRS+HIPEC has been confirmed by several studies (5) and is considered one of several acceptable options for women with stage 3 EOC by NCCN experts at present. However, there are few high-quality clinical trials comparing conventional debulking surgery $v s$. CRS+HIPEC to treat the long-term outcomes of advanced EOC patients.

This study applies a propensity score matching (PSM) analysis method to eliminate confounding factors from clinicopathological variables to compare the efficacies between conventional debulking surgery and CRS+HIPEC in patients with EOCPC. We present the following article in accordance with the STROBE reporting checklist (available at https://dx.doi.org/10.21037/tcr-20-3233).

\section{Methods}

\section{Study population}

A total of 200 ovarian cancer patients with FIGO stage III/ IV underwent surgeries at the Department of Peritoneal Cancer Surgery in Beijing Shijitan Hospital from May 2004 to November 2019. Patients were divided into a conventional debulking surgery group (the control group) and a CRS+HIPEC group (the study group) according to the initial treatments by diagnosis. Patients with any of the following features were excluded from this analysis: (I) follow-up $<1$ month and (II) diagnosis of nonepithelial ovarian cancer, such as ovarian germ cell carcinoma, ovarian gonadal sex cord stromal tumor, and metastasis, including Krukenberg tumor and other clinicopathological types. Our final study population included 186 patients (Figure 1).

The study was conducted in accordance with the Declaration of Helsinki (as revised in 2013) and the Harmonized Tripartite Guideline for Good Clinical Practice from the International Conference on Harmonization. All patients enrolled completed this informed consent form. All data in this retrospective study were analyzed anonymously, and the study was approved by the Beijing Shijitan Hospital ethics board [(approve number is 2019(07)].

\section{Debulking surgery procedures}

The traditional therapy strategy for advanced EOC is primary debulking surgery combined with platinumand taxane-derived intravenous chemotherapy (IV) and intraperitoneal chemotherapy (IP) $(6,7)$. The scope of the surgery included hysterectomy with bilateral adnexectomy + greater omentum resection + appendectomy (for mucinous carcinoma) + pelvic lymph node dissection + para-aortic lymph node dissection. Optimal debulking surgery was defined based on the criteria of the Gynecologic Oncology Group (GOG). For residual disease (RD), RD0 indicates no macroscopic residual disease after debulking surgery; $\mathrm{RD} 1$ indicates that the largest residual tumor is $\leq 1 \mathrm{~cm}$; and RD2 indicates residual tumor $>1.0 \mathrm{~cm}$. Both RD0 and RD1 represent complete debulking. Patients with RD2 are considered to have incomplete debulking. The postoperative adjuvant therapies were at the discretion of the treating physicians, and these treatment strategies include IV, IP, IV and IP chemotherapies; targeted therapies such as bevacizumab and apatinib; and pelvic radiotherapy for patients with pelvic lymph node metastasis and/or large tumor residuals.

\section{CRS+HIPEC procedures}

Full abdominal exploration was performed through a midline xiphoid-pubic incision after general anesthesia to record the invasion degree, ascites, volume and location of the primary tumor and the peritoneal cancer index (PCI). The maximal CRSs were performed. The extent of CRS was determined by Sugarbaker's criteria on the complete cytoreductive (CC) surgery score. CC0 indicates no residual peritoneal disease after CRS; CC1, residual disease $\leq 2.5 \mathrm{~mm}$ in diameter; CC2, residual tumor between $2.5 \mathrm{~mm}$ and $2.5 \mathrm{~cm}$; and $\mathrm{CC} 3$, residual tumor $>2.5 \mathrm{~cm}$ in diameter or the presence of a sheet of unresectable tumor nodules (8).

After CRS, open HIPEC was implemented with cisplatin $120 \mathrm{mg}+$ mitomycin C $30 \mathrm{mg}$ or docetaxel/paclitaxel $120 \mathrm{mg}+$ cisplatin $30 \mathrm{mg}$, each dissolved in $3,000 \mathrm{~mL}$ of heated saline at $43{ }^{\circ} \mathrm{C}$ for $60 \mathrm{~min}$. Then, gastrointestinal anastomoses or stomata were made. Patients received adjuvant chemotherapy after the operation, including IV and IP.

\section{Follow-up}

All patients were regularly followed up once every 


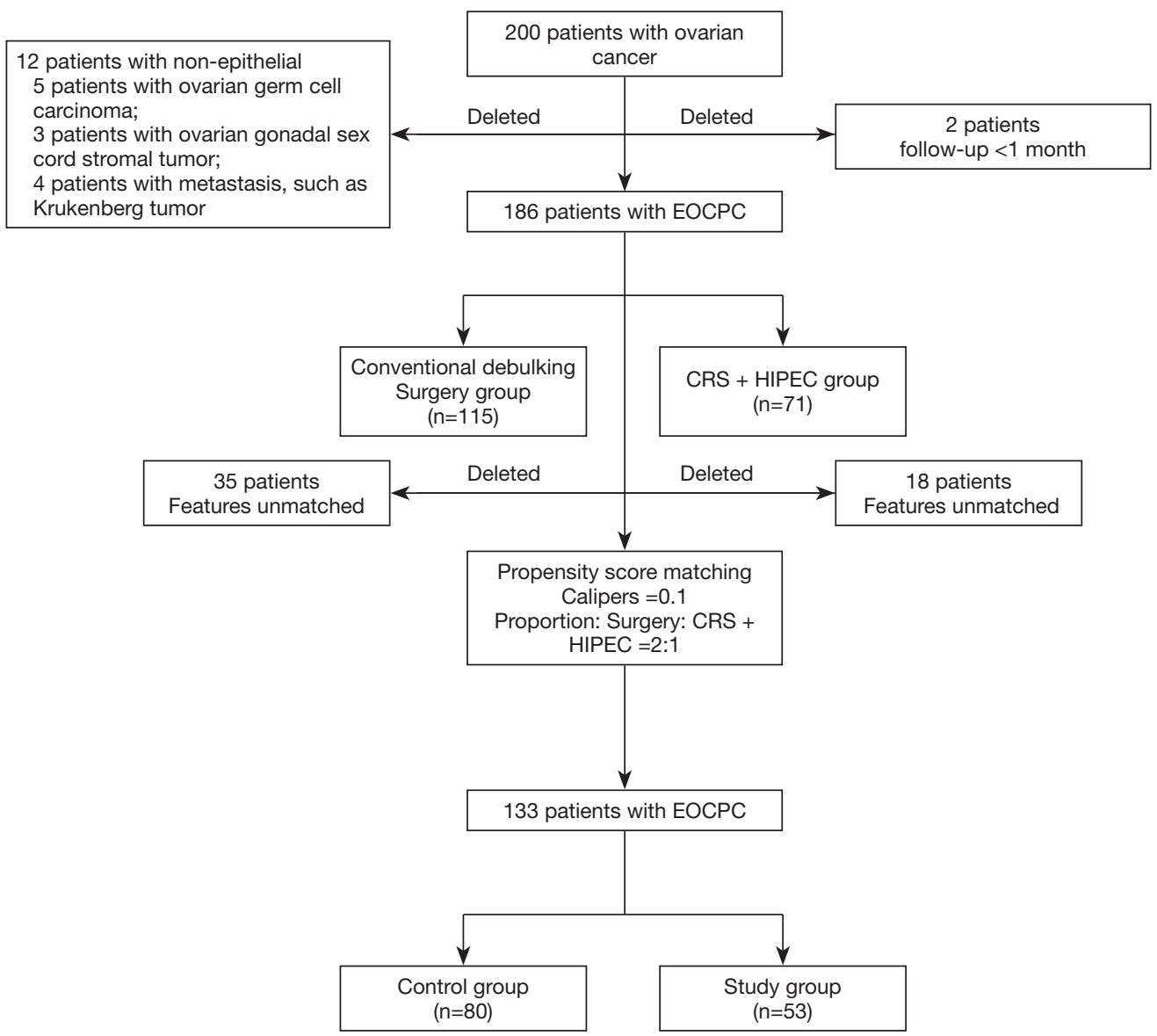

Figure 1 Flow chart of patient selection. Of 200 patients, 14 patients with non-epithelial ovarian cancer and follow-up $<1$ month were excluded, and the remaining 186 patients were matched. Patients were assigned to the study and control groups according to the initial treatment after diagnosis. The two treatment strategies were compared for efficacy.

3 months for the first 2 years, every 6 months for years 3 to 5 , and every year thereafter for detailed information on disease status. The follow-up package included physical examination, serum tumor marker levels including carbohydrate antigen 125 (CA 125, normal range: 0$35 \mathrm{U} / \mathrm{mL}$ ), carbohydrate antigen 199 (CA 199, normal range: $0-37 \mathrm{U} / \mathrm{mL}$ ), and carcinoembryonic antigen (CEA, normal range: $0-5 \mathrm{ng} / \mathrm{mL}$ ), and imaging examination, with the most recent follow-up on November 30, 2019.

The primary endpoints of this study were overall survival (OS) and progression-free survival (PFS). OS was defined as the time interval from the first surgery to tumorrelated death or the last follow-up. PFS was calculated from the date of surgery until the last follow-up, including the following criteria: first, the patients who underwent surgery-based curative comprehensive treatment developed any clinical manifestations; second, the CA 125 level rose again after surgery; third, the medical imaging discovered any mass in the operation field; and finally, the biopsy confirmed the diagnosis.

\section{Statistical analysis}

Data analysis including PSM was performed with IBM SPSS Statistics 24, R version 3.1.0 \& PS Matching package version 3.04 according to Thoemmes et al. (9). To reduce the variability and heterogeneity among the patients and to reduce the selection bias, a PSM model was applied to calculate the following covariates: age, performance status (KPS) score, histopathology, tumor differentiation, chemotherapy, radiotherapy, targeted therapy, and immunotherapy. Patients treated with CRS+HIPEC were matched at a ratio of $1: 2$ with patients treated with conventional debulking surgery using the nearest neighbor 
method based on the PSM with a caliper of 0.1 .

Continuous variables were reported as medians (interquartile ranges) and compared with the MannWhitney $\mathrm{U}$ test after the study for skewness, as appropriate. Categorical variables were expressed as proportions and percentages and were compared with univariate analysis, the $\chi^{2}$ test and Fisher's exact method. Survival analysis is shown as the median value with a $95 \%$ confidence interval and was compared with the log-rank test.

\section{Results}

\section{Baseline characteristics of the patients}

The median follow-up was 19.9 (1.8-121.5) months, and no patients were lost. Before matching, a total of 186 EOCPC patients with FIGO stage IIIC and IV disease were included, with 71 patients $(38.2 \%)$ who underwent CRS+HIPEC (the study group) and 115 patients $(61.8 \%)$ who underwent conventional debulking surgery (the control group) in the initial treatment. The median age was 58 years (22-87 years), the median KPS was 80 [50-100], and the median PCI was 19 [1-39]. According to the classification of the histological types, 163 patients had serious carcinoma (87.6\%), 10 patients had mucinous carcinoma (5.4\%), and 13 patients had other types $(7.0 \%)$. For the tumor differentiations, 20 cases (10.8\%) were well differentiated, while 166 cases $(89.2 \%)$ had intermediatepoor differentiation. The baseline parameters of the patients are shown in Table 1. Before matching, the KPS score in the study group was significantly lower than that in the control group $(\mathrm{P}=0.015)$. The numbers of cycles in the $\mathrm{IV}(\mathrm{P}=0.002)$ and radiotherapy $(\mathrm{P}=0.031)$ groups were significantly less than those in the control group. Moreover, the differences in tumor differentiation between the two groups were significant $(\mathrm{P}=0.028)$. To reduce heterogeneity and selection bias, the PSM model was calculated at a ratio of 2:1. There were no differences in KPS score, tumor differentiation, number of IVs or radiotherapy between the two groups after matching $(\mathrm{P}>0.05)$ (Table 1, Figure $\mathrm{S} 1)$.

\section{Overall survival}

After matching at a ratio of 2:1, a total of 133 patients were included for the final analysis, which was assigned to $80(60.2 \%)$ patients in the control group and $53(39.8 \%)$ patients in the study group. At the end of the study, 9
$(9 / 53,17.0 \%)$ patients were deceased, and 44 (44/53, $83.0 \%)$ patients were alive in the study group. Meanwhile, $28(28 / 80,35.0 \%)$ patients were deceased and $52(52 / 80$, $65.0 \%$ ) patients were alive in the control group (Figure $2 A$ ), with significant differences in survival rate between the two groups $(\mathrm{P}=0.02)$. The median OS was 87.3 months $[95 \%$ confidence interval (CI), not reached] in the study group, which was significantly longer than that in the control group [25.2 months (95\% CI, 21.7-28.6, P=0.002 with the logrank test)] (Figure 2B). The 1-, 2-, 3-, 4-, and 5-year survival rates in the study group vs. the control group were $88.3 \%$ vs. $73.2 \%, 76.4 \%$ vs. $59.8 \%, 70.0 \%$ vs. $36.7 \%, 70.0 \%$ vs. $36.7 \%$ and $46.7 \%$ vs. $18.3 \%$, respectively (Figure $2 C$ ). The 5 -year survival rate in the study group was 2.5 times that of the control group ( $46.7 \%$ vs. $18.3 \%$, respectively, $\mathrm{P}=0.003$ ), and the 3-and 4-year survival rates were 1.9 times that of the control group ( $70.0 \%$ vs. $36.7 \%$, respectively, $\mathrm{P}=0.016$ ), though the 1- and 2-year survival rates were similar between the two groups $(\mathrm{P}>0.05)$.

\section{Subgroup analysis of patients who underwent optimal/ complete CRS}

Patients who achieved CC0-1 in CRS+HIPEC or RD01 in conventional debulking surgery were defined as having complete cytoreduction. After matching, CC01 was achieved in $42(42 / 53,79.2 \%)$ patients in the study group, and $13(13 / 42,31.0 \%)$ had recurrence. RD 0-1 was achieved in $59(59 / 80,73.7 \%)$ patients in the control group, and $26(26 / 59,44.1 \%)$ had recurrence. The recurrence rate between the two groups was significantly different $(\mathrm{P}=0.035)$ (Figure 3A). The median PFS was 19.6 months (95\% CI, 12.6-26.5 months) in the study group, which was significantly longer than that in the control group [10.1 months (95\% CI, 7.6-12.7 months, $\mathrm{P}=0.007$ with the log-rank test)] (Figure 3B).

\section{Subgroup analysis of the patients who underwent incomplete CRS}

CC 2-3 in CRS+HIPEC and RD2 in conventional debulking surgery represented incomplete CRS. After matching, 11 $(11 / 53,20.8 \%)$ patients were CC2-3 in the study group, and $21(21 / 80,26.3 \%)$ patients were RD2 in the control group; however, there were no differences between the study and control groups in regard to OS (25.9 vs. 12.1 months, respectively, $\mathrm{P}=0.082$ ). The median $\mathrm{OS}$ of $\mathrm{CC} 0-1$ was 
Table 1 Demographic and baseline characteristics of patients

\begin{tabular}{|c|c|c|c|c|c|c|c|c|}
\hline Parameters & $\mathrm{n}$ & \multicolumn{2}{|c|}{ Before PSM } & $P$ value & no. & \multicolumn{2}{|c|}{ After PSM } & $P$ value \\
\hline Total no. & 186 & 71 & 115 & & 133 & 53 & 80 & \\
\hline Age (year) & & & & 0.140 & & & & 1.000 \\
\hline$\leq 58$ & 94 & 31 & 63 & & 65 & 26 & 39 & \\
\hline Histological type & & & & 0.118 & & & & 0.943 \\
\hline Serous & 163 & 65 & 98 & & 124 & 49 & 75 & \\
\hline Mucous & 10 & 1 & 9 & & 2 & 1 & 1 & \\
\hline Others & 13 & 5 & 8 & & 7 & 3 & 4 & \\
\hline Intermediate-poor differentiation & 166 & 68 & 98 & & 8 & 3 & 5 & \\
\hline KPS score & & & & 0.015 & & & & 0.217 \\
\hline$\leq 80$ & 105 & 32 & 73 & & 67 & 23 & 44 & \\
\hline$>80$ & 81 & 39 & 42 & & 66 & 30 & 36 & \\
\hline IV & & & & 0.002 & & & & 1.000 \\
\hline Yes & 177 & 63 & 114 & & 131 & 52 & 79 & \\
\hline No & 9 & 8 & 1 & & 2 & 1 & 1 & \\
\hline IP & & & & 0.364 & & & & 1.000 \\
\hline Radiotherapy & & & & 0.031 & & & & 1.000 \\
\hline Yes & 170 & 69 & 101 & & 130 & 52 & 78 & \\
\hline No & 16 & 2 & 14 & & 3 & 1 & 2 & \\
\hline Targeted therapy & & & & 0.580 & & & & 0.677 \\
\hline Yes & 148 & 55 & 93 & & 102 & 42 & 60 & \\
\hline No & 38 & 16 & 22 & & 31 & 11 & 20 & \\
\hline Immunotherapy & & & & 0.744 & & & & 1.000 \\
\hline Yes & 176 & 68 & 108 & & 126 & 50 & 76 & \\
\hline No & 10 & 3 & 7 & & 7 & 3 & 4 & \\
\hline
\end{tabular}

PSM, propensity score matching; KPS, Karnofsky's performance scoring; IV, intravenous chemotherapy; IP, intraperitoneal chemotherapy. 

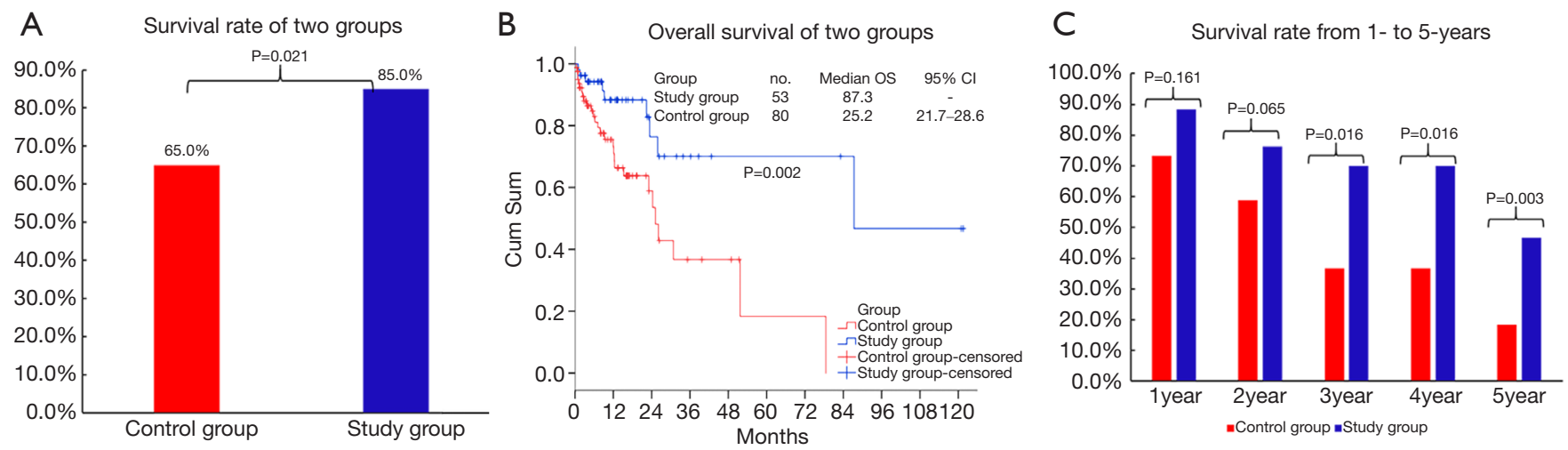

Figure 2 Overall survival. (A) Survival rate between the two groups; (B) median overall survival between the two groups; (C) the 1- to 5 year survival rates in the study group were $88.3 \%, 76.4 \%, 70.0 \%, 70.0 \%$, and $46.7 \%$, respectively, while they were $73.2 \%, 58.9 \%, 36.7 \%$, $36.7 \%$, and $18.3 \%$ in the control group, showing significant differences between the two groups.
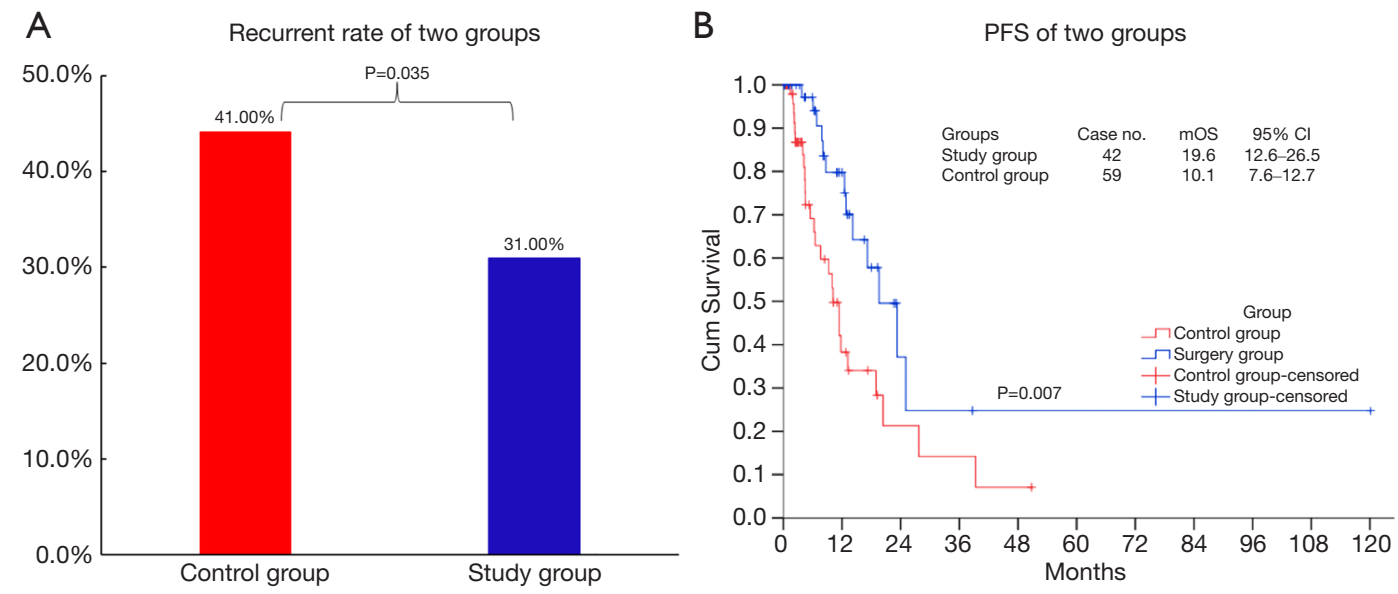

Figure 3 Progression-free survival analysis. (A) There was a significant difference in the recurrence rate between the two groups (P=0.035). (B) The median PFS rates were 19.6 months (95\% CI, 12.6-26.5 months) in the study group and 10.1 months (95\% CI, 7.6-12.7) in the control group $(\mathrm{P}=0.007)$.

103.3 months (95\% CI, 86.0-120.7 C months) in the study group, which was significantly longer than that of RD $0-1$ in the control group [ 46.2 months (95\% CI, 30.0-62.3 months, $\mathrm{P}=0.020$ with the log-rank test)] (Figure 4).

\section{Discussion}

Our study provides data from patients who were assigned to undergo CRS+HIPEC and conventional debulking surgery for the initial therapy strategy of advanced EOC. The current studies focused on CRS+HIPEC are mostly limited to retrospective cohorts or single-group trials, are heterogeneous with regard to combined inclusions of primary and recurrent diseases and lack unbiased data.

Here, we adopted PSM to analyze the data, the purpose of which was to validate the technique as a tool for balancing the baseline variables between two groups. It is crucial to include all known variables and confounders that may impact treatment allocation and postmatching analysis to demonstrate a very balanced population. At the beginning of this study, univariate analysis was conducted to quantify preoperative heterogeneity, which built the basis of our PSM method. Moreover, a matched data set ratio of 2:1 was utilized to measure tumor differentiation, KPS score, number of IVs and radiotherapy in the two groups. Finally, OS and PFS analyses based on CC were conducted. 
A

A Overall Survival of Complete Cytoreduction

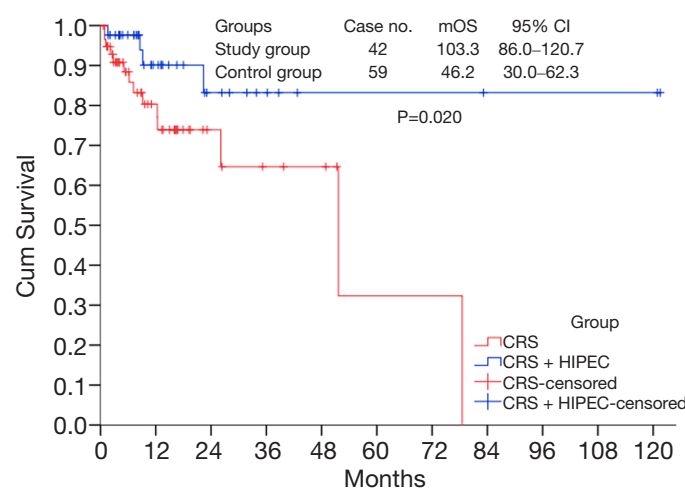

B

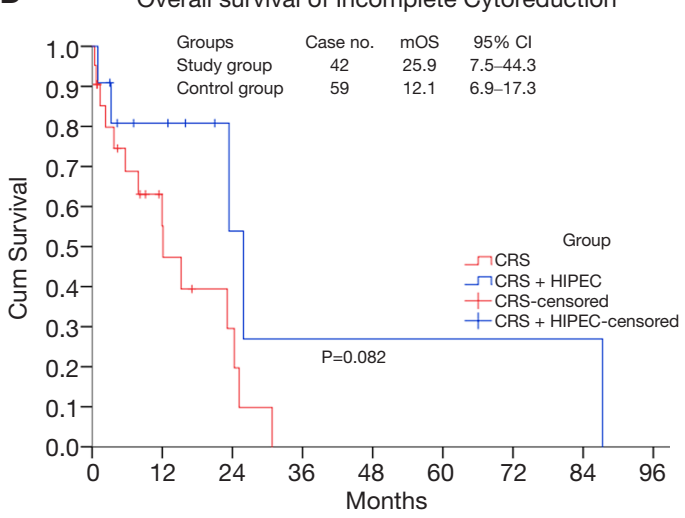

Figure 4 OS analysis in patients in the two groups who underwent complete and incomplete cytoreduction. (A) The median OS rates in the CC 0-1 and R0-1 groups. (B) The median OS rates in the CC 2-3 and RD2 groups. OS, overall survival.

After excluding confounding factors, the efficacies of CRS+HIPEC and conventional debulking surgery were analyzed and discussed.

In essence, by comparing long-term survival, we were able to evaluate whether CRS+HIPEC, as a comprehensive therapy strategy, justifies the routine treatment of advanced EOC. The results showed that the OS in CRS+HIPEC was 87.3 months (over 7 years), which was longer than that in conventional debulking surgery. However, the survival rate in CRS+HIPEC was $18 \%$ higher than that in conventional debulking surgery. The 3-and 4-year survival rates of CRS+HIPEC were 1.9 times higher than those of conventional debulking surgery $(70.0 \%$ vs. $36.7 \%$, respectively), and the 5 -year survival rate was 2.5 times higher than that of conventional debulking surgery $(46.7 \%$ vs. $18.3 \%$, respectively), which was an increase of $28.4 \%$. All of these results indicated that patients with advanced EOC who underwent CRS+HIPEC could achieve a higher survival rate than conventional debulking surgery.

The more extensive surgery to minimize tumor burden is the determination of the success of CRS+HIPEC and conventional debulking surgery, which is an independent prognostic factor for patients with advanced EOC. Because of the different excision extensions from CRS+HIPEC and conventional debulking surgery, the criteria of evaluation for complete CRS were also different $(2,10)$. To compare the OS and PFS from different surgery strategies, subgroup analysis was performed after matching. The results showed that the recurrence rate of $\mathrm{CC} 0$ patients who underwent CRS+HIPEC was 1.4 times lower than that of RD 0-1 patients who underwent conventional debulking surgery (31.0\% vs. $44.1 \%$, respectively), which was a decrease of $13.1 \%$. Moreover, the median PFS in CRS+HIPEC was 9.5 months longer than that in conventional debulking surgery. OS was also determined between the two therapies and was defined as incomplete CRS for patients who did not reach CC 0-1 and RD 0-1. Additionally, the subgroup analysis showed that the median OS in CRS+HIPEC was 87.3 months (over 7 years) (Figure $4 A, B$ ), which was longer than that in conventional debulking surgery. It was further verified that CRS+HIPEC has a long-term survival benefit for patients and has become an indispensable treatment measure in both widespread metastasis and late-stage tumors.

Since 1980, many studies have reported that complete CRS is combined with platinum- and taxane-based systemic chemotherapy as the standard first-line treatment of advanced EOC (11-13). By undergoing this mode of treatment, the natural course of advanced EOC was 22.346.4 months, the 5 -year survival rate was approximately $30 \%, 75 \%$ of patients relapsed after the initial traditional treatment strategy $(14,15)$, and the median PFS was 6.1 to 20.0 months $(11-13,16-18)$. However, the median OS in CRS+HIPEC was 22.0 to 74.0 months, the 5 -year survival rate was $24.5 \%$ to $56.5 \%$, the mortality rate was $22.0 \%$ to $43.0 \%$, and the median PFS was $12.5-57.5$ months for patients with recurrence after initial treatments $(1,19-31)$ (Table 2). Of special note, the OS and PFS in our study were significantly better than those reported in the literature, which was supposed to be the main reason why 


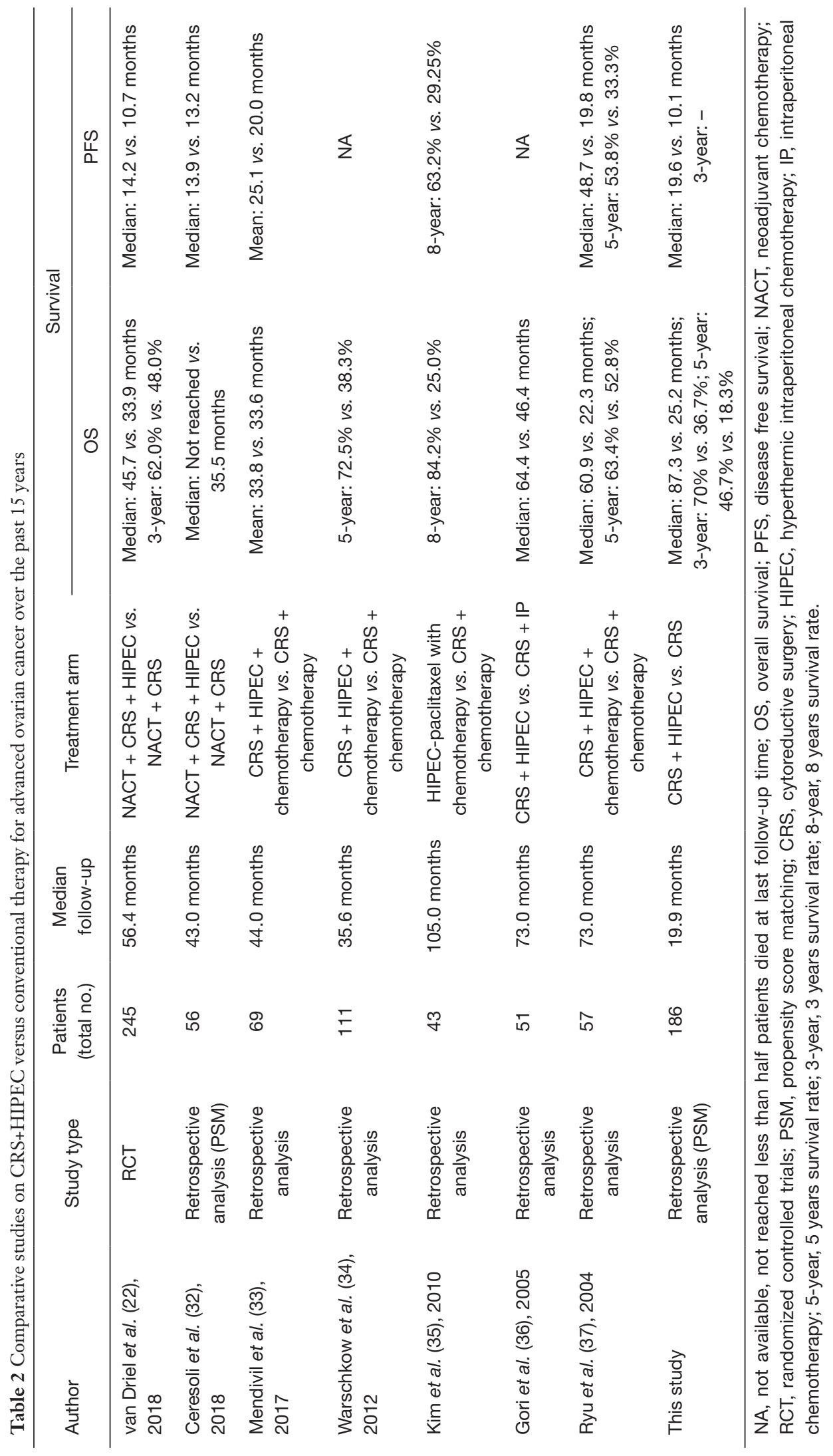


our research group focused on CRS+HIPEC, which is a new comprehensive prevention and treatment technology for PC, to investigate preclinical and clinical studies on the feasibility, efficacy, and safety of this multidisciplinary treatment approach in animal models and clinical settings since 2003. Standardized surgery and procedures could be helpful to achieve strict CC0, which is an independent prognostic index for PC patients.

In the current study, we evaluated HIPEC as a single IP administration strategy during surgery, which improved the distribution of heated chemotherapy in the abdominal cavity, enhanced the survival rate and reduced the recurrence rate.

\section{Conclusions}

In summary, our findings have confirmed that there are more advantages of CRS+HIPEC in the OS and PFS of patients with advanced EOC than standard treatments. Although the PSM balances baseline data of variables in two groups, the strength of the evidence is still weak in a randomized controlled study. These results have accurately demonstrated the clinical practice that CRS+HIPEC is an indispensable comprehensive treatment strategy for patients with advanced EOC. In addition, the standardized operation and evaluation system is able to provide distinctive survival benefits.

\section{Acknowledgments}

Thanks for the support of Chinese Fournal of Clinical Oncology (a domestic journal of China), in which a part of data from this article was published on November, 2019, issue 49 (6): 994-998. With the permission and authorization of the journal, we updated the data and translated it into English and submitted to the journal of Translational Cancer Research. Funding: Supported by the grants supporting Beijing Municipal Administration of Hospitals' Ascent Plan (DFL20180701), Special Fund for the Capital Characteristic Clinical Medicine Development Project (Z161100000516077), Beijing Municipal Grant for Medical Talents Group on Peritoneal Surface Oncology (2017400003235J007), Key Discipline Development Fund of Beijing Shijitan Hospital affiliated to the Capital Medical University (2016fmzlwk), Beijing Natural Science Foundation (7172108), Health Science Promotion Project of Beijing (2018-TG-27), Hubei Provincial Natural Science Foundation of China (2017CFB177).

\section{Footnote}

Reporting Checklist: The authors have completed the STROBE reporting checklist. Available at https://dx.doi. org/10.21037/tcr-20-3233

Data Sharing Statement: Available at https://dx.doi. org/10.21037/tcr-20-3233

Conflicts of Interest: All authors have completed the ICMJE uniform disclosure form (available at https://dx.doi. org/10.21037/tcr-20-3233). The authors have no conflicts of interest to declare.

Ethical Statement: The authors are accountable for all aspects of the work in ensuring that questions related to the accuracy or integrity of any part of the work are appropriately investigated and resolved. The study was conducted in accordance with the Declaration of Helsinki (as revised in 2013) and the Harmonized Tripartite Guideline for Good Clinical Practice from the International Conference on Harmonization. All patients enrolled completed the informed consent form. All data in the retrospective study were analyzed anonymously which approved by Beijing Shijitan Hospital ethic board [(approve number is 2019(07)].

Open Access Statement: This is an Open Access article distributed in accordance with the Creative Commons Attribution-NonCommercial-NoDerivs 4.0 International License (CC BY-NC-ND 4.0), which permits the noncommercial replication and distribution of the article with the strict proviso that no changes or edits are made and the original work is properly cited (including links to both the formal publication through the relevant DOI and the license). See: https://creativecommons.org/licenses/by-nc-nd/4.0/.

\section{References}

1. Kireeva GS, Gafton GI, Guseynov KD, et al. HIPEC in patients with primary advanced ovarian cancer: Is there a role? A systematic review of short- and long-term outcomes. Surg Oncol 2018;27:251-8.

2. Hennessy BT, Coleman RL, Markman M. Ovarian cancer. Lancet 2009;374:1371-82.

3. Goh J, Mohan GR, Ladwa R, et al. Frontline treatment of epithelial ovarian cancer. Asia Pac J Clin Oncol 2015;11 Suppl 6:1-16. 
4. Cascales-Campos P, López-López V, Gil J, et al. Hyperthermic intraperitoneal chemotherapy with paclitaxel or cisplatin in patients with stage III-C/IV ovarian cancer. Is there any difference? Surg Oncol 2016;25:164-70.

5. Baiocchi G, Ferreira FO, Mantoan H, et al. Hyperthermic Intraperitoneal Chemotherapy after Secondary Cytoreduction in Epithelial Ovarian Cancer: A Single-center Comparative Analysis. Ann Surg Oncol 2016;23:1294-301.

6. Komiyama S, Kato K, Inokuchi Y, et al. Bevacizumab combined with platinum-taxane chemotherapy as first-line treatment for advanced ovarian cancer: a prospective observational study of safety and efficacy in Japanese patients (JGOG3022 trial). Int J Clin Oncol 2019;24:103-14.

7. Kireeva GS, Bespalov VG, Belyaeva OA, et al. Normothermic and Hyperthermic Intraperitoneal Chemoperfusions with Cisplatin to Treat Advanced Ovarian Cancer in Experimental Settings. Pathol Oncol Res 2017;23:459-60.

8. Halkia E, Spiliotis J, Sugarbaker P. Diagnosis and management of peritoneal metastases from ovarian cancer. Gastroenterol Res Pract 2012;2012:541842.

9. West SG, Cham H, Thoemmes F, et al. Propensity scores as a basis for equating groups: basic principles and application in clinical treatment outcome research. J Consult Clin Psychol 2014;82:906-19.

10. Sugarbaker PH. Cytoreductive surgery and perioperative intraperitoneal chemotherapy for the treatment of advanced primary and recurrent ovarian cancer. Curr Opin Obstet Gynecol 2009;21:15-24.

11. 11. DiSilvestro P, Alvarez Secord A. Maintenance treatment of recurrent ovarian cancer: Is it ready for prime time? Cancer Treat Rev 2018;69:53-65.

12. Aschenbrenner DS. New Maintenance Treatment for Recurrent Ovarian, Fallopian Tube, or Primary Peritoneal Cancer. Am J Nurs 2018;118:25-6.

13. Coleman RL, Brady MF, Herzog TJ, et al. Bevacizumab and paclitaxel-carboplatin chemotherapy and secondary cytoreduction in recurrent, platinum-sensitive ovarian cancer (NRG Oncology/Gynecologic Oncology Group study GOG-0213): a multicentre, open-label, randomised, phase 3 trial. Lancet Oncol 2017;18:779-91.

14. Armstrong DK, Bundy B, Wenzel L, et al. Intraperitoneal cisplatin and paclitaxel in ovarian cancer. $\mathrm{N}$ Engl J Med 2006;354:34-43.

15. Nasu K, Kai K, Hirakawa T, et al. Retrospective analysis of outcomes of secondary debulking surgery for recurrent epithelial ovarian cancer with favorable prognostic factors. J Obstet Gynaecol Res 2014;40:791-6.

16. Provencher DM, Gallagher CJ, Parulekar WR, et al. OV21/PETROC: a randomized Gynecologic Cancer Intergroup phase II study of intraperitoneal versus intravenous chemotherapy following neoadjuvant chemotherapy and optimal debulking surgery in epithelial ovarian cancer. Ann Oncol 2018;29:431-8.

17. Mueller JJ, Kelly A, Zhou Q, et al. Intraperitoneal chemotherapy after interval debulking surgery for advanced-stage ovarian cancer: Feasibility and outcomes at a comprehensive cancer center. Gynecol Oncol 2016;143:496-503.

18. Ren Y, Shi T, Jiang R, et al. Multiple Cycles of Neoadjuvant Chemotherapy Associated With Poor Survival in Bulky Stage IIIC and IV Ovarian Cancer. Int J Gynecol Cancer 2015;25:1398-404.

19. Petrillo M, De Iaco P, Cianci S, et al. Long-Term Survival for Platinum-Sensitive Recurrent Ovarian Cancer Patients Treated with Secondary Cytoreductive Surgery Plus Hyperthermic Intraperitoneal Chemotherapy (HIPEC). Ann Surg Oncol 2016;23:1660-5.

20. Cowan RA, O'Cearbhaill RE, Zivanovic O, et al. Current status and future prospects of hyperthermic intraoperative intraperitoneal chemotherapy (HIPEC) clinical trials in ovarian cancer. Int J Hyperthermia 2017;33:548-53.

21. Arjona-Sanchez A, Rufián-Peña S. Progress in the management of primary and recurrent ovarian carcinomatosis with peritonectomy procedure and HIPEC in a high volume centre. Int J Hyperthermia 2017;33:554-61.

22. van Driel WJ, Koole SN, Sikorska K, et al. Hyperthermic Intraperitoneal Chemotherapy in Ovarian Cancer. N Engl J Med 2018;378:230-40.

23. Wang Y, Ren F, Chen P, et al. Effects of CytoReductive surgery plus hyperthermic IntraPEritoneal chemotherapy (HIPEC) versus CytoReductive surgery for ovarian cancer patients: A systematic review and meta-analysis. Eur J Surg Oncol 2019;45:301-9.

24. Cascales-Campos PA, Gil J, Gil E, et al. Treatment of microscopic disease with hyperthermic intraoperative intraperitoneal chemotherapy after complete cytoreduction improves disease-free survival in patients with stage IIIC/ IV ovarian cancer. Ann Surg Oncol 2014;21:2383-9.

25. Cascales Campos P, Gil J, Parrilla P. Morbidity and mortality outcomes of cytoreductive surgery and hyperthermic intraperitoneal chemotherapy in patients 
with primary and recurrent advanced ovarian cancer. Eur J Surg Oncol 2014;40:970-5.

26. Malgras B, Gayat E, Aoun O, et al. Impact of Combination Chemotherapy in Peritoneal Mesothelioma Hyperthermic Intraperitoneal Chemotherapy (HIPEC): The RENAPE Study. Ann Surg Oncol 2018;25:3271-9.

27. Manzanedo I, Pereira F, Pérez-Viejo E, et al. Hyperthermic intraoperative intraperitoneal chemotherapy (HIPEC) with primary or secondary cytoreductive surgery in the treatment of advanced epithelial ovarian cancer. Minerva Ginecol 2017;69:119-27.

28. Di Giorgio A, De Iaco P, De Simone M, et al. Cytoreduction (Peritonectomy Procedures) Combined with Hyperthermic Intraperitoneal Chemotherapy (HIPEC) in Advanced Ovarian Cancer: Retrospective Italian Multicenter Observational Study of 511 Cases. Ann Surg Oncol 2017;24:914-22.

29. Cavaliere D, Cirocchi R, Coccolini F, et al. 1st Evidencebased Italian consensus conference on cytoreductive surgery and hyperthermic intraperitoneal chemotherapy for peritoneal carcinosis from ovarian cancer. Tumori 2017;103:525-36.

30. Sun JH, Ji ZH, Yu Y, et al. Cytoreductive Surgery plus Hyperthermic Intraperitoneal Chemotherapy to Treat Advanced/Recurrent Epithelial Ovarian Cancer: Results from a Retrospective Study on Prospectively Established Database. Transl Oncol 2016;9:130-8.

31. Lim MC, Kang S, Choi J, et al. Hyperthermic intraperitoneal chemotherapy after extensive cytoreductive surgery in patients with primary advanced epithelial ovarian cancer: interim analysis of a phase II study. Ann Surg Oncol 2009;16:993-1000.

32. Ceresoli M, Verrengia A, Montori G, et al. Effect of cytoreductive surgery and hyperthermic intraperitoneal chemotherapy on relapse pattern in primary epithelial ovarian cancer: a propensity score based case-control study. J Gynecol Oncol 2018;29:e53.

33. Mendivil AA, Rettenmaier MA, Abaid LN, et al. Consolidation hyperthermic intraperitoneal chemotherapy for the treatment of advanced stage ovarian carcinoma: a 3 year experience. Cancer Chemother Pharmacol 2017;80:405-10.

34. Warschkow R, Tarantino I, Lange J, et al. Does hyperthermic intraoperative chemotherapy lead to improved outcomes in patients with ovarian cancer? A single center cohort study in 111 consecutive patients. Patient Saf Surg 2012;6:12.

35. Kim JH, Lee JM, Ryu KS, et al. Consolidation hyperthermic intraperitoneal chemotherapy using paclitaxel in patients with epithelial ovarian cancer. J Surg Oncol 2010;101:149-55.

36. Gori J, Castaño R, Toziano M, et al. Intraperitoneal hyperthermic chemotherapy in ovarian cancer. Int J Gynecol Cancer 2005;15:233-9.

37. Ryu KS, Kim JH, Ko HS, et al. Effects of intraperitoneal hyperthermic chemotherapy in ovarian cancer. Gynecol Oncol 2004;94:325-32.
Cite this article as: Zhang J, Li XB, Ma R, Ji ZH, Bai W, Li Y. Long term survival of cytoreductive surgery plus hyperthermic intraperitoneal chemotherapy in advanced epithelial ovarian cancer. Transl Cancer Res 2021;10(8):3705-3715. doi: 10.21037/ tcr-20-3233 


\section{Supplementary}

A

B

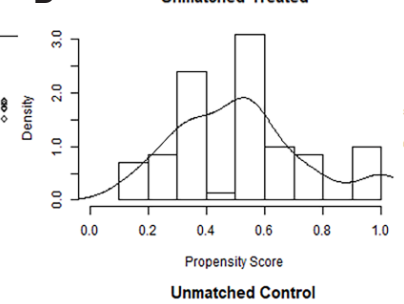

Unmatched Control

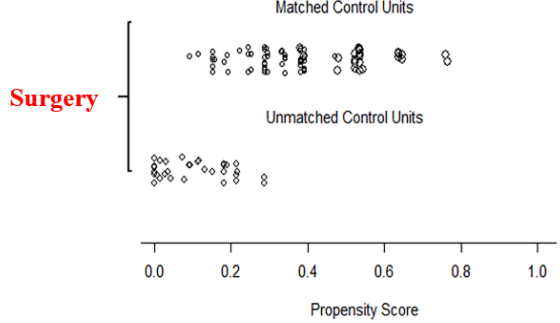

Distribution of Propensity Scores

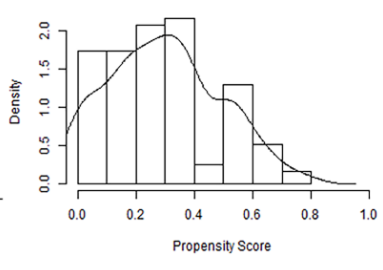

Matched Treated
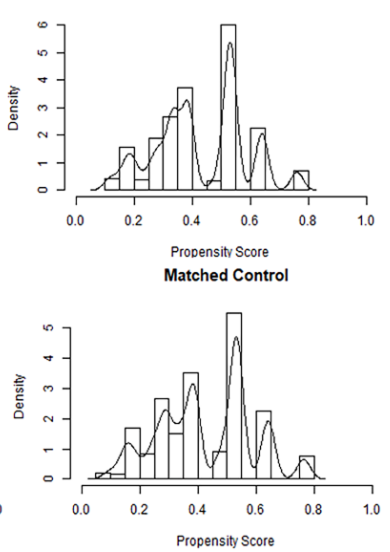

C standardized differences before matchin!

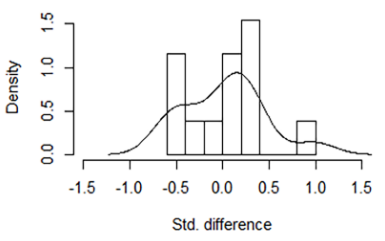

Standardized differences after matching

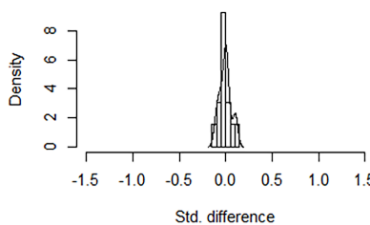

Figure S1 Jitter plot of propensity score matching (PSM). (A) Scatter diagram of PSM. Data were skewed distributed before PSM but were balanced after matching. (B) Histogram of PSM. The data distribution in the two groups was heterogeneous before PSM but homogeneous after matching. (C) Histogram of standardized differences. Large discreteness of data before PSM but were concentrated after matching. 